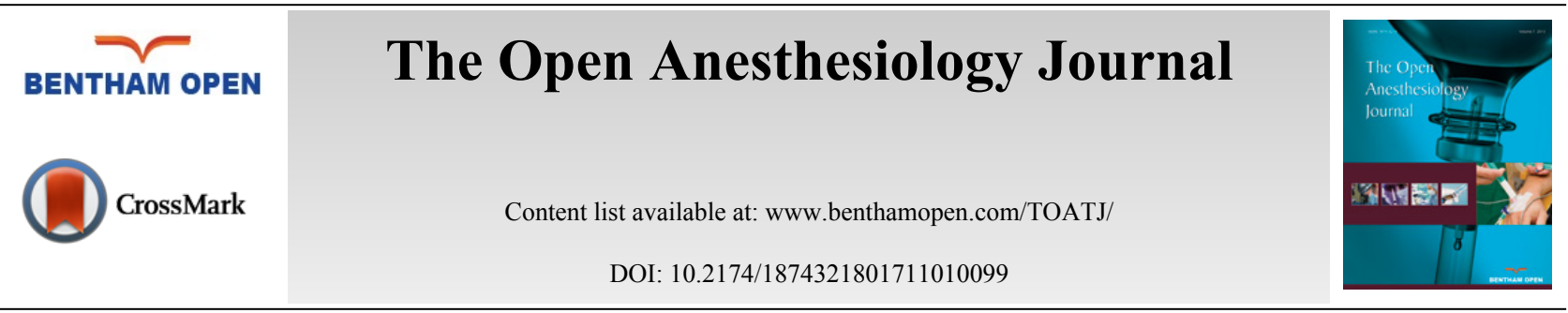

BOOK REVIEW

\title{
Book Review: Basic Anesthesiology Examination Review
}

\author{
Michael G Irwin * and Ka Ying Chow \\ Department of Anaesthesiology, Queen Mary Hospital, University of Hong Kong, Hong Kong
}

This book is very useful for basic exam preparation and we actually evaluated it for this purpose using several trainees who were taking the examination for Fellowship of the Hong Kong College of Anaesthesiologists and for the Australia and New Zealand College (the exams are separate but very similar in structure and content).

It comprises of 32 chapters characterized into 6 sections covering all essential topics included in the outline of the Basic Anesthesiology Examination of the American Board of Anesthesiology. The authors aim to provide exam candidates with a focused resource for preparation. Questions and answers are included in each chapter and this helps to bring each chapter together and tests overall understanding.

The book precisely covers a wide range of contents including basic science, Anatomy, Clinical Science, Pharmacology, Statistics and Basic Anesthesia Equipment and Monitoring. The content is thorough and, since it is tailored to the outline of the Basic Anesthesiology Examination, candidates who know this material well should be able to answer most of the exam questions that I have seen in the past papers. Although focused on North American practice, it will also be useful for the Australasian and European examinations.

Section 1: Anatomy. The information included here is quite basic and there is not much diagramatic illustration. This is a slight drawback as readers may find it difficult to visualise and memorise the actual anatomy. It is more of an overview of the topic and descriptions do not include fine details. However, it is useful in the exam setting when you only have limited time to read a few short paragraphs. Anatomy questions are often difficult to handle, but this section provides a good example on how to describe the anatomy in a concise manner.

Section 2: Physics, Monitoring, and Anesthesia Delivery Devices. This chapter would also be very useful for new anaesthesia trainees. The complex anaesthetic delivery systems are made easy to understand which is very important since a good understanding is absolutely necessary for anaesthetists to practise safely, and respond to and manage crisis situations. This is a really strong chapter as we had difficulty finding legible material on this topic until this book was published.

Section 3: Principles of Pharmacology. A systematic explanation of crucial pharmacological principles. Drug information is well oraganized and presented and even the newest drugs are included. This section is valuable to all anaesthetists for rapid review and assimilation of large amounts of complex information about pharmacology.

Section 4: Clinical Sciences. Very good overview of most important anaesthetic procedures. Basic anaesthetic trainees can definitely be benefited from reading this section. It could be improved by adding in clinical pictures or drawings for better illustration. However, it is highly ranked for the purpose of tackling exam questions.

Section 5: Organ-based Basic and Clinical Sciences. Physiology has always been one of the most challenging topics for anaesthetists. This section provides an easily comprehensible explanation of various organ systems. Useful diagrams are included to assist understanding. It is highly recommended for both initial learning and last minute exam preparation.

\footnotetext{
* Address correspondence to this author at the Department of Anaesthesiology, Queen Mary Hospital, University of Hong Kong, Hong Kong; E-mail: mgirwin@hku.hk
} 
Overall this book is broad in scope and yet concise which is difficult to achieve. Important concepts and information are clearly highlighted. It was, and still considered excellent for quick, final revision immediately before the exam (all our trainees passed!). We would also recommend it to new anaesthetic trainees who want to get an overview of anaesthesia when they start their training. However, it should not be regarded as a primary study resource. References and further reading are included in the end of each chapter. Readers should go through the suggested references for a deeper understanding of each topic in order to be able to apply the knowledge to their daily anaesthetic practice for best tailored patient care.

(C) 2017 Irwin and Chow.

This is an open access article distributed under the terms of the Creative Commons Attribution 4.0 International Public License (CC-BY 4.0), a copy of which is available at: (https://creativecommons.org/licenses/by/4.0/legalcode). This license permits unrestricted use, distribution, and reproduction in any medium, provided the original author and source are credited. 